\title{
Investigation of Drift Rate of Solar Radio Burst Type II Due To Coronal Mass Ejections Phenomenon
}

\author{
N.H. Zainol ${ }^{1}$, Z. S. Hamidi ${ }^{1 *}$, N. N. M. Shariff ${ }^{2}$, S. Arifin ${ }^{1}$, C. Monstein ${ }^{3}$ \\ ${ }^{1}$ School of Physics and Material Sciences, Faculty of Sciences, MARA University of Technology, \\ 40450, Shah Alam, Selangor, Malaysia \\ ${ }^{2}$ Academy of Contemporary Islamic Studies (ACIS), MARA University of Technology, \\ 40450, Shah Alam, Selangor, Malaysia \\ ${ }^{3}$ Institute of Astronomy, Wolfgang-Pauli-Strasse 27, Building HIT, Floor J, \\ $\mathrm{CH}-8093$ Zurich, Switzerland \\ *E-mail address: zetysh@salam.uitm.edu.my
}

\begin{abstract}
The formation of detected solar radio burst type II occurred was captured using Compound Astronomical Low Cost Frequency Spectrometer Transportable Observatory (CALLISTO) system which gives a better resolution of a wonderful image than other countries. The phenomenon was found on $2^{\text {nd }}$ November 2014 at 09:39 [UT] in Switzerland. CALLISTO spectrometer device detects and traces a Coronal Mass Ejections (CMEs) phenomenon that causes the occurrence of the solar burst type II. As it happened, the drift rate of the solar radio burst Type II is calculated and discussed in details. Plasma frequency $\left(f_{p}\right)$, Langmuir waves and type II radiation relates each other in the establishment of this phenomenon. This paper presents a study of drift rate selected event of solar radio burst type II based on CMEs. The drift rate at this moment was about $3.2 \mathrm{MHz} / \mathrm{s}$ which has low drift rate thus the velocity OF THE CMEs was just about $695 \mathrm{~km} / \mathrm{s}$ shown from NOAA.
\end{abstract}

Keywords: Sun; low frequency; solar radio; burst; type II; e-CALLISTO

\section{INTRODUCTION}

In radio astronomy, solar radio emission the observations of solar bursts by low spectral and spatial resolution instruments can provide the light curve and a crude spectrum of the whole flares and Coronal Mass Ejections which may consist of many distinct sources with different characteristics [1]. Sun contains of ionized gases such as $92.1 \%$ of hydrogen gas and $7.8 \%$ of helium gas mixed up and held together by its own gravity [2]. The different parts of the sun structure rotate at different rates since it does not have a rigid surface. Sun can be divided into 6 parts of its structure which contains (i) core, (ii) radiation zone, (iii) convection zone, (iv) photosphere, (v) chromospheres and (iv) corona [3]. Figure 1 shows the main structure of the Sun. 


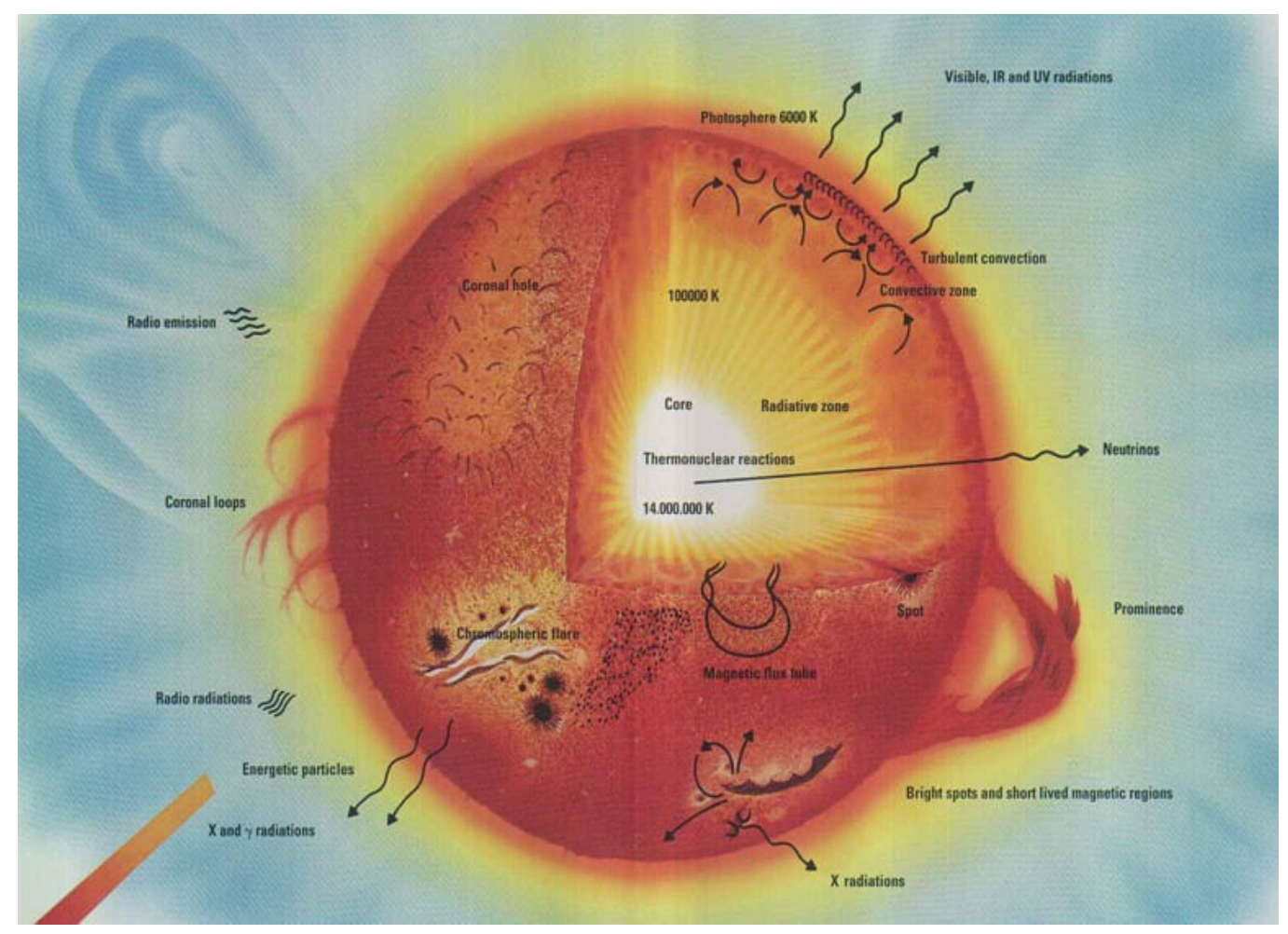

Figure 1. Sun Structures.

Sun's core has immense pressure and its temperature is about 15 million degrees Celsius which enough for thermonuclear fusion to occur[4]. The reaction will produce energy in the core that powers the sun and produce essential heat and light to the Earth [5]. This energy will be carried by the radiation outward to the radiative zone and proceed to convective zone where large bubbles of hot plasma move upwards and the temperature drops below 2 million degrees Celsius by taking about 170000 years for energy to travelled from the core to the convective zone. Photosphere region (5,500 degrees Celsius) is where the radiation escapes outward and the sunspots phenomenon occurs. The numbers of sunspot fluctuate every 11 years and as part of sun's magnetic activity cycle [6].

\section{CORONAL MASS EJECTIONS AND DRIFT RATE OF TYPE II SOLAR BURST}

The cycles also connected with bright solar flares and Coronal Mass Ejections (CMEs) that blast off the Sun [7]. Light from the orange red layer of chromosphere and corona layer lie on photosphere usually too weak to be seen against the brighter photosphere. The temperature increases with high altitude above the photosphere reaching two million degrees Celsius. However, the source of coronal heating has been a scientific mystery since more than 50 years ago. Shocks generated by CMEs can be inferred from type II radio bursts in the corona and in the IP medium which are termed as metric and interplanetary type II bursts, respectively [8]. Coronal Mass Ejection or called as CME is one of the event that causes of solar burst and being classified as Type II [9]. It is an observable change in coronal structure that occurs on a time scale either in a few minutes or several hours. It can be classified as a massive burst of solar wind and magnetic fields rising above the solar corona that can trigger 
major disturbances in Earth's magnetosphere [10]. The speed of CMEs may not be much higher than $3000 \mathrm{~km} / \mathrm{s}$, consistent with the free energy available in active regions [11]. CMEs causes a huge plasma clouds produced by plasma emission mechanism to leave the Sun which means it ejects large scale density waves converted into escaping radio waves out into space and after a few hours or days, it may enters the Earth and cause geomagnetic storm [12]. Theoretically, time variability in the emission may due to the changes in the electron density [13]. Basically, solar radio burst was used to determine solar activity and level or radiation that received from the Sun [14]. Type II is slow drift from high to low frequency in dynamic radio spectra burst where it is more comparative rare and the rate of occurrence also small. The occurrence of this event is rare. However, the frequency emission may drift over hundreds of megacycles per second. Normally, the burst begins suddenly with fundamental and second harmonic beginning almost simultaneously [15]. Figure 2 shows the event detected by CALLISTO spectrometer at low frequency. For us to know, the frequency drift rate of solar burst is a power law in the frequency of emission. Drift rate $(d f / d t)$ is a displacement of the peak in frequency per unit time. The absolute value of rate decreases with decreasing in frequency. It is determined by taking the start time to end time and start frequency to the end frequency of the solar burst Type II seen in Figure I. Drift rate of the solar radio burst type II was defined as (equation 1):

$$
\text { Dirft Rate, } \frac{d f}{d t}=\frac{\left(f_{\varepsilon}-f_{s}\right)}{\left(t_{\varepsilon}-t_{s}\right)}\left(\frac{M H z}{s}\right)
$$

Where $f_{e}$ is frequency of end time, $f_{s}$ is frequency of start time, $t_{e}$ is end time, $t_{s}$ start time.

This power law was also valid when observations from different spectral domains [metric (m), decameter-hectometric (DH), and kilometric $(\mathrm{km})$ wavelengths] are combined for the same events or for different events. Similarly, the power law was maintained when observations from different instruments and different epochs are combined. This universal nature of the power law suggests that the same shock should be involved in producing radio bursts at various spectral domains. The energy distribution for type II is within range of $1.506 \times 10^{-6} \mathrm{eV}$ to $6.906 \times 10^{-6} \mathrm{eV}$. Dynamic spectra of solar radio burst type II discovered as slowly drifting bands, often in pairs differing in frequency by a factor $\approx 2$. They were quickly interpreted in terms of coronal shock wave accelerating electrons, driving Langmuir waves near the electron plasma frequency $f_{p}$ and $2 f_{p}$. This type II bursts was definitely incorporates with Coronal Mass Ejections (CMEs), travelling shock waves, reflected electron, Langmuir waves and radiation approaches $f_{p}$ and $2 f_{p}$. Inner corona of Sun presents an evolution of the CMEs. It undergoes a rapid acceleration in the beginning, after it starts from static as it erupts and reaches a maximum acceleration within the inner corona before being controlled by the aerodynamic drag. Langmuir waves starts from a disturbance (plasma) in the form of a longitudinal (electrostatic wave) that propagates in the plasma due to variations in the plasma's electron density. Specifically, Langmuir waves are collective oscillations of inhomogeneous bunches of electrons displaced from their natural equilibrium, in which the inertia of the relatively massive ions serves to establish an electrostatic restoring force that tries to bring the electrons back to their equilibrium positions. 


\section{METHODOLOGIES}

CALLISTO spectrometer is a programmable receiver built to observe or detect solar radio burst that occur at each time and particular location [16]. The main applications of CALLISTO programme are observation of solar radio bursts and Radio Frequency Interference (RFI)-monitoring for astronomical science, education and others [17]. The instrument operates between 45 to $870 \mathrm{MHz}$ using a modern, commercially available broadband cable-TV tuner having a frequency resolution of $62.5 \mathrm{KHz}$. Based on the data, Type II burst occurred within ten minutes [18]. The data are transferred via a cable to a computer and saved locally. Time resolution is $0.25 \mathrm{sec}$ at 200 channels per spectrum (800 pixels per second) [19]. The overall dynamic range is larger than $50 \mathrm{~dB}$. Many CALLISTO instruments have already been deployed in India, Switzerland, United Kingdom, Russia, South Africa, Australia, Malaysia, Indonesia and many more [20]. Callisto in addition is constructed to do radio-monitoring within its frequency range .

The solar radio emission as observed from ground-based telescopes at meter and decimeter wavelengths poses still has many challenges and potential treasures after many decades of study [21,22]. When examining the result or data using the Callisto spectrometer device, there are two types of Type II burst pattern which is (i) harmonic structure and (ii) herring-bone structure (doubling both fundamental bands results in thick band). Appearance of Herring-born structure leads in which slow drifting band of Type II burst appears to be a source from which rapidly drifting elements diverge towards lower and higher frequency. Since the local plasma frequency decreases with radial distance away from the Sun, the plasma frequency in the radio-emitting region of the shock decreases with time [23]. So, the emission of the shock drifts to lower frequencies with time at the local plasma frequency. Thus, if the density variation in the source path was known, the frequency drift rate of type II solar radio burst can be converted into the speed of the radio source.

\section{RESULT AND ANALYSIS}

The CMEs speed needs to exceed the characteristic speed before it can produces a shock, and the combination of CME acceleration and the rapid change in the fast mode speed produces possibilities for shock formation and decay. The drift rate frequency of type II bursts in the solar radio dynamic spectra are related to the speed of the shock that produces the bursts and the density gradient in the ambient medium. By using equation 1, drift rate calculated was $-3.2 \mathrm{MHz} / \mathrm{s}$. The starting time taken was at 9:42UT with frequency of $80 \mathrm{MHz}$ to end time 9:52 UT with frequency of $48 \mathrm{MHz}$ 


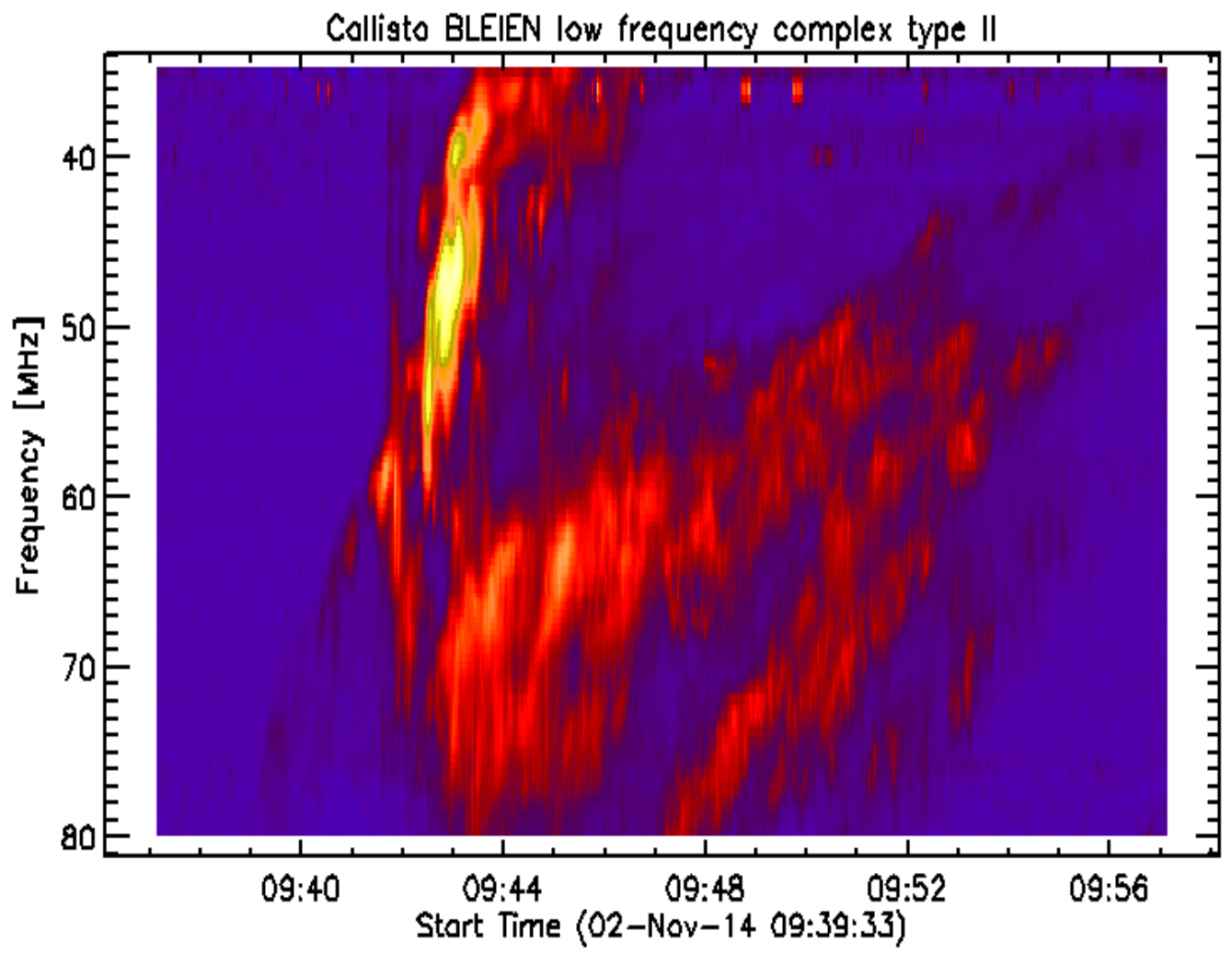

Figure 2. Solar burst Type II (Herring-bone structure) detected in Switzerland on $2^{\text {nd }}$ November 2014 at 9:42 (UT).

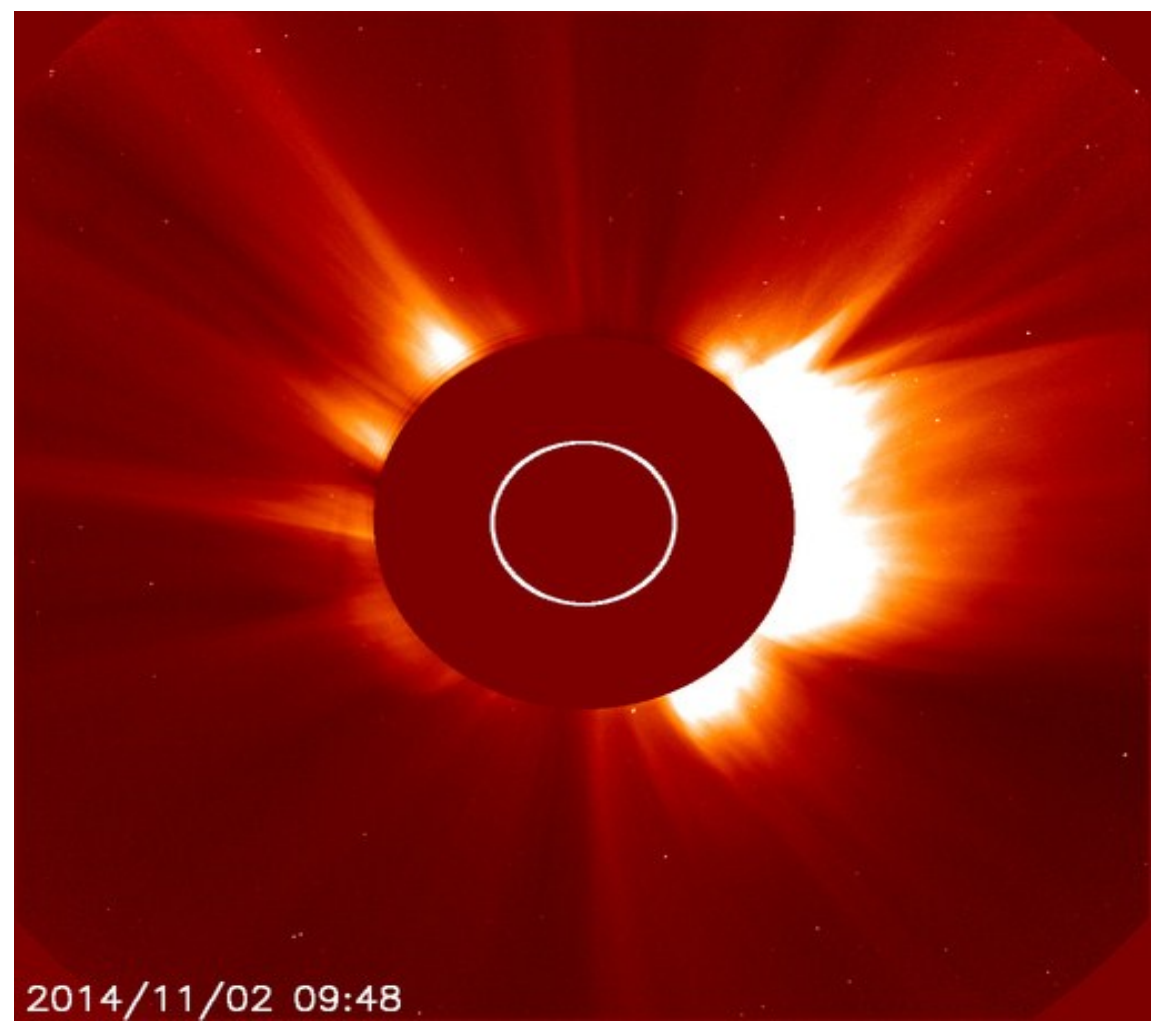

Figure 3. Large Angle and Spectrometric Coronagraph (LASCO) detection of CMEs. 
Based on Figure 2, the upper pattern is harmonic pattern (fundamental pattern) while the bottom one is Herring-bone pattern. This paper only discussed on Herring-bone pattern. The 'Herringbones' are rapidly drifting emission stripes resembling type II radio burst and seem to be started at the 'backbones'. It generates the Type II burst which is interpreted as the radio signature of electrons accelerated shock wave. During this event, this explosion, the solar plasma is heated to tens of millions degrees, protons and electrons, with nuclei accelerated to near the speed of light. The superheated electrons from CMEs move along the magnetic field lines faster than solar wind can flow. They will be accelerated by CMEs driven shock at very great energies throughout the interplanetary (IP) medium. Based on Figure 3, the explosion was observed by LASCO coronagraph. The structure of exploitation and their instability condition is not easy to be understood. However, there is an indication of fast pulsations with a period of sub-second time that contributes to this event.

The CME height is to be the same as the shock height and the type II burst height. This is strictly not true because the shock is expected to be located ahead of the CME and the type II burst is located at the shock front. The frequency drift rate of the burst is related to the shock's velocity. The slow drift rate determines the lifespan for the particular solar radio burst type II. With a slow drift rate of 3.2 MHz/s, it has only short lifespan. The exciter of the type II burst is a shock wave (independent of any shock which may or may not have been associated with the earlier transient mass motion itself) which is generated at the site of and simultaneous with the impulsive phase of flare. Fast and wide CMEs do not drive shocs or they drive weak shocks that do not produce detectable radio emission. The X-ray region observation has a lot of advantages in the sense of observing the solar activity. This short wavelength can provide a starting point of the eruption. Then, it can show the major percentage of energy of the explosions. The CMEs characteristics in x-ray regions can provide a finding at early stage of the phenomenon as it has a short wavelength in the electromagnetic spectrum.

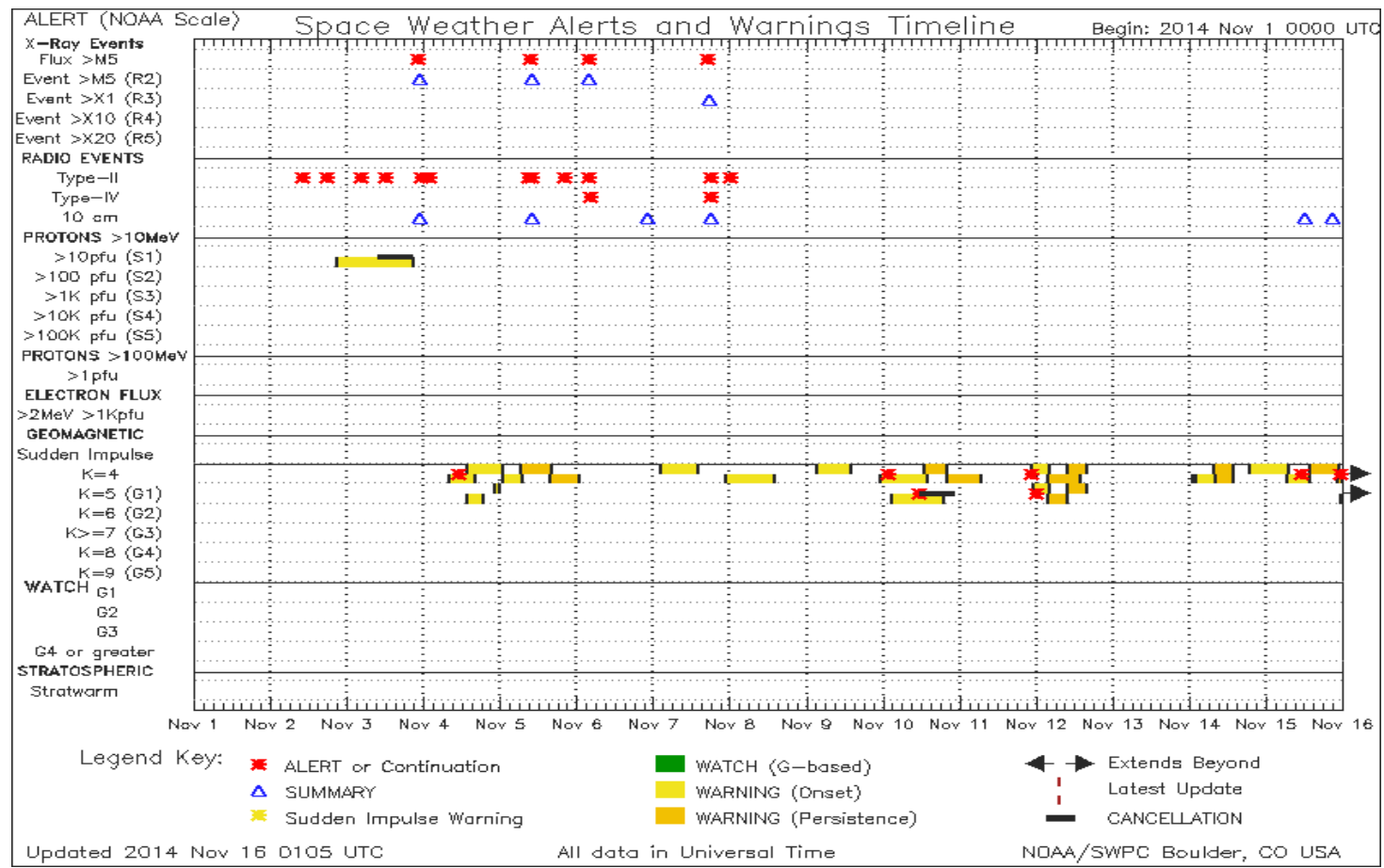

Figure 4. X-ray region of Space Weather Alert and Warning Timeline from National Oceanic and Atmospheric Administration (NOAA). 
Based on Figure 4, there was an alert alarming on $2^{\text {nd }}$ November 2014 where shows that there was something obstruction happened in space. Details from National Oceanic and Atmospheric Administration (NOAA) were starts with Space Weather Message Code: ALTTP2, Serial Number: 973 which issued at 2014 Nov 021019 UTC. Then, more details includes were ALERT: Type II Radio Emission which begin on 2014 Nov 020941 UTC with estimated velocity of $695 \mathrm{~km} / \mathrm{s}$. With this detail, solar radio burst Type II emissions occur in association with eruptions on the sun and typically indicate a coronal mass ejection is associated with a flare event. Without the benefit of hard X-ray or gamma-ray observations, we were not able to make an assessment of the role of energetic particles in the event. The core events were distinguished with the same time but different place taken by CALLISTO spectrometer in India (Figure 5a) and United Kingdom (Figure 5b).
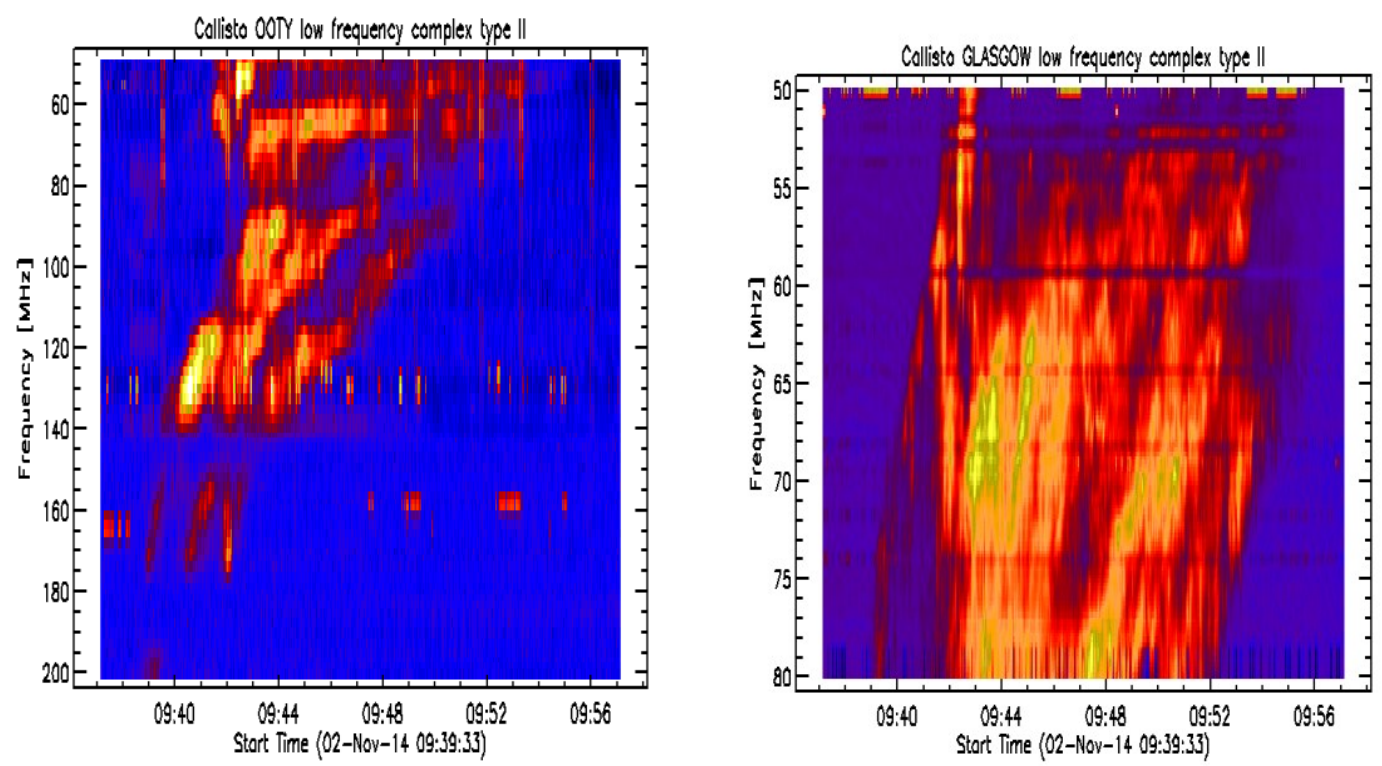

Figure 5. (a) Result taken in India (b) Result obtained in Glasgow.

The solar burst type II taken in India appears the fundamental structure pattern while in Glasgow, United Kingdom, harmonic pattern appeared. Both occurred due to Coronal Mass Ejections (CMEs).

\section{CONCLUSION}

Solar radio burst type II due to Coronal Mass Ejections (CMEs) was detected on $2^{\text {nd }}$ November 2014 in Switzerland by CALLISTO spectrometer as detectable device. The drift rate at this moment was about $3.2 \mathrm{MHz} / \mathrm{s}$ which has low drift rate thus the velocity OF THE CMEs was just about $695 \mathrm{~km} / \mathrm{s}$ shown from NOAA. With a low drift rate will leads to low velocity of the event to occur. 


\section{Acknowledgement}

We are grateful to CALLISTO network, STEREO, LASCO,SDO/AIA, NOAA and SWPC make their data available online. This work was partially supported by the 600-RMI/FRGS 5/3 (135/2014) and 600RMI/RAGS 5/3 (121/2014) UiTM grants and Kemenetrian Pengajian Tinggi Malaysia. Special thanks to the National Space Agency and the National Space Centre for giving us a site to setup this project and support this project. Solar burst monitoring is a project of cooperation between the Institute of Astronomy, ETH Zurich, and FHNW Windisch, Switzerland, MARA University of Technology and University of Malaya. This paper also used NOAA Space Weather Prediction Centre (SWPC) for the sunspot, radio flux and solar flare data for comparison purpose. The research has made use of the National Space Centre Facility and a part of an initiative of the International Space Weather Initiative (ISWI) program.

\section{Biography}

Nur Hidayah Zainol is an undergraduate student at the School of Physics and Material Sciences, Faculty of Sciences, MARA University of Technology, 40450, Shah Alam, Selangor, Malaysia.

Dr Zety Sharizat Hamidi is currently a lecturer and focused in Solar Astrophysics research specifically in radio astrophysics at the School of Physics and Material Sciences, Faculty of Sciences, MARA University of Technology, 40450, Shah Alam, Selangor, Malaysia. Involve a project under the International Space Weather Initiative (ISWI) and also a lecturer in School of Physics and Material Science, at MARA University of Technology, Shah Alam Selangor.

C.Monstein is a senior Engineer at Institute of Astronomy, Wolfgang-Pauli-Strasse 27, Building HIT, Floor J, CH-8093 Zurich, Switzerland and one of the researchers who initiated the CALLISTO system around the world.

Dr Nur Nafhatun Md Shariff is a senior lecturer in Academy of Contemporary Islamic Studies (ACIS), MARA University of Technology, 40450, Shah Alam, Selangor, Malaysia.Her current research is more on sustainability; environmental aspect. She is looking forward for cross-field research, i.e. solar astrophysics, light pollution measurement (mapping) and religious studies.

\section{References}

[1] Z. S. Hamidi1, N. N. M. Shariff2, C. Monstein3, W. N. A. Wan Zulkifli1,, N.S.A. M. B. Ibrahim1, N. A. Amran1, Investigation on a Broken Solar Burst Type II during High Activities in AR1613 on 13th November 2012, (2014).

[2] M.R. Kundu, Solar Radio Astronomy, John Wiley, 1965.

[3] Nelson, a. Melrose, Solar Radiophysics Cambridge Univ. Press, New York, 1985.

[4] W. Kundu, Solar and Space Weather Radiophysics, Dordrecht: Kluwer, 2004.

[5] M.V. Goldman, D.F. Smith, Physics of the Sun, Dordrecht: Reidel, 1986.

[6] D.J. McLean, a.N.R. Labrum, Solar Radiophysics, Cambridge University Press, Cambridge, 1985.

[7] A.G. Emslie, H. Kucharek, B.R. Dennis, N. Gopalswamy, G.D. Holman, G.H. Share, A. Vourlidas, T.G. Forbes, P.T. Gallagher, G.M. Mason, T.R. Metcalf, R.A. Mewaldt, R.J. Murphy, R.A. Schwartz, T.H. Zurbuchen, Energy partition in two solar flare//CME events, Journal of Geophysical Research 109 (2004) 15.

[8] A. Mujiber Rahman, S. Umapathy, A. Shanmugaraju, Y.J. Moon, Solar and interplanetary parameters of CMEs with and without type II radio bursts, Advances in Space Research 50 (2012) 516-525. 
[9] N. Gopalswamy, et al., Relation between type II bursts and CMEs inferred from STEREO observations, Sol. Phys. 259 (2009b) 227-254.

[10] T.C. G. Mann, H. Aurab, Characteristics of coronal shock waves and solar type II radio bursts, (1994).

[11] N. Gopalswamy, Coronal mass ejections and other extreme characteristics of the 2003 October-November solar eruptions, Journal of Geophysical Research 110 (2005).

[12] K.A. Marsh, Hurford, G.J, Ann. Rev. Astron. Astrophysics 20 (1982) 497.

[13] Z. S. Hamidi1, C. Monstein2, N. N. M. Shariff 3, Radio Observation of Coronal Mass Ejections (CMEs) Due to Flare Related Phenomenon on 7th March 2012, International Letters of Chemistry, Physics and Astronomy (2014).

[14] N. Gopalswamy, Radio-rich Solar Eruptive Events, Geophys. Res. Lett 27 (2000).

[15] Z. Hamidi, N. Shariff, C. Monstein, Fundamental and Second Harmonic Bands of Solar Radio Burst Type II Caused by X1. 8-Class Solar Flares, (2014).

[16] Z. Hamidi, N. Shariff, C. Monstein, Comparison of the Radio Frequency Interference (RFI) in the Region of Solar Burst Type III Data At Selected CALLISTO Network, International Letters of Chemistry, Physics and Astronomy 10 (2014) 38-45.

[17] Z. Hamidi, N. Shariff, C. Monstein, Evaluation of Spectral Overview and Radio Frequency Interference (RFI) Sources at Four Different Sites in CALLISTO Network at the Narrow Band Solar Monitoring Region, (2014).

[18] Z. Hamidi, N. Shariff, C. Monstein, Type II Solar Radio Burst with a Split and HerringBones During a Minimum Solar Activity, International Letters of Chemistry, Physics and Astronomy 13 (2014) 104-111.

[19] Z. Hamidi, N. Shariff, C. Monstein, First Light Detection of A Single Solar Radio Burst Type III Due To Solar Flare Event, (2014).

[20] Z. Hamidi, N. Shariff, Z. Abidin, Z. Ibrahim, C. Monstein, Coverage of Solar Radio Spectrum in Malaysia and Spectral Overview of Radio Frequency Interference (RFI) by Using CALLISTO Spectrometer from $1 \mathrm{MHz}$ to $900 \mathrm{MHz}$, Middle-East Journal of Scientific Research 12 (2012) 893-898.

[21] A.O. Benz, C. Monstein, H. Meyer, P.K. Manoharan, R. Ramesh, A. Altyntsev, A. Lara, J. Paez, K.S. Cho, A World-Wide Net of Solar Radio Spectrometers: e-CALLISTO, Earth, Moon, and Planets 104 (2009) 277-285.

[22] Z. Hamidi, N. Shariff, C. Monstein, The Different Between the Temperature of the Solar Burst at the Feed Point of the Log Periodic Dipole Antenna (LPDA) and the CALLISTO Spectrometer, (2014).

[23] N.G. E. Agullar-Rodriguez, R. MacDowall, S. Yashiro, M. L. Kaiser, A Study Of The Drift Rate of Type II Radio Burst At Different Wavelength, (2005). 